\title{
EL LENGUAJE DE LAS MANOS \\ EN LA DOCENCIA UNIVERSITARIA. ANÁLISIS DE LAS ACTITUDES PROYECTADAS EN EL ALUMNADO
}

\author{
MARÍA HERNÁNDEZ HERRARTE \\ Universidad Europea Miguel de Cervantes
}

\section{RESUMEN}

Esta investigación analiza el papel que juegan las manos y sus movimientos en el discurso del docente universitario a la hora de transmitir conocimientos teóricos. Partimos de la hipótesis de que aquellos educadores que utilizan sus manos de forma más efectiva son los que transmiten actitudes más positivas en los estudiantes eliminando barreras en la comunicación no verbal que dificultan el desarrollo de las actividades del aula. La metodología se basa en un análisis de frecuencias y se completa con un cuestionario realizado por alumnos universitarios. Los resultados permiten afirmar que aquellos docentes con movimientos de manos más acertados son aquellos que se perciben con actitudes más positivas.

PALABRAS CLAVE: comunicación no verbal, lenguaje de las manos, docencia universitaria, proyección de actitudes, análisis de frecuencias.

\section{ABSTRACT}

The present article analyses both the communicative hand-gesturing and the object-directed hand movement in the teaching process of theoretical contents among faculty. Our hypothesis sets that: hand gestures and object-directed hand movement in faculty is directly related to a more efficient conveyance of positive attitudes; and these two kinetic aids also help overcome communication barriers that might hedge classroom activities. The methodology involved an ad hoc screening sheet, based on a frequency analysis for a number of variables, complemented with the answers extracted from a questionnaire distributed among senior year students. Results proved our hypothesis true, as faculty with a more efficient hand-gesturing and object-directed hand movement in their teaching were perceived with more positive attitudes.

KEYWORDS: nonverbal communication, hand-gesturing, university teaching, conveyance of attitudes, frequency analysis. 


\section{INTRODUCCIÓN Y ESTADO DE LA CUESTIÓN}

Las manos y sus movimientos son los protagonistas de este estudio cuyo principal objetivo se materializa en descubrir las actitudes que los docentes universitarios proyectan en sus alumnos a través de este potente instrumento de comunicación no verbal. Y es que el lenguaje de las manos ayuda al profesor a ilustrar su mensaje, apoyándole o contradiciéndole. Las manos son un indicador del estado emocional, motivo por el que aquel comunicador que mejor las utilice será el que consiga persuadir mejor (Arellano, 2006: 4). Los movimientos manuales comunican contenidos de lo más variado: apoyan lo que se dice, lo adelantan o se expresan por sí mismos (González, 2003: 62). También indican la tensión del individuo, y son una expresión personal y única del estilo de cada uno (Villahizán Pérez, 2007: 155 y Esteve, 2006: 86).

La hipótesis de partida de este estudio se basa en que las manos son una de las partes del cuerpo con mayor potencial comunicativo y podría enunciarse de la siguiente manera: aquellos profesores universitarios que realizan gestos con sus manos asociados a parámetros positivos son valorados por sus alumnos como más comunicativos, con mayor capacidad para hacerse entender, más empáticos, más cercanos y más dinámicos; son actitudes que sin duda favorecen el proceso de aprendizaje en el aula. Por el contrario, los profesores con movimientos de manos menos acertados son percibidos como más tímidos, distantes, antipáticos y con mayores dificultades para hacerse entender, aspectos que dificultan la adquisición de competencias por parte de los estudiantes.

Bien, pues una vez definida la hipótesis y el objetivo del trabajo, pasamos a continuación a una revisión de las investigaciones teóricas nacionales e internacionales relacionadas con este campo de estudio.

El ser humano puede realizar hasta 700.000 signos diferentes combinando los movimientos del brazo, muñeca y dedos de las manos (Cáceres, 2003: 199). Por eso los gestos manuales sirven para ilustrar o subrayar lo que se dice, para señalar distancias y direcciones o para indicar formas, tamaños o movimientos (Cruz Díaz, 2003: 188). Pero en el contexto de la docencia universitaria, es importante subrayar que la utilización de las manos sirve para captar la atención, aumentar el impacto de la comunicación y ayudar a retener mejor la información (Pease, 2006: 143).

Los movimientos manuales son distintos en cada individuo, siendo su relevancia variable. Se pueden encontrar tres grandes grupos: los de manejo, como coger o mover cosas de sitio (no tienen un objetivo comunicacional), las señales orientadas a la relación y los gestos relacionados con el discurso, que transmiten mensajes sobre la cuestión tratada (Neill y Caswell, 2005: 30). Estos últimos son los que más interesan en este estudio. Cuando un profesor se encuentra delante de sus alumnos, sus manos serán 
una de sus principales fuentes de expresión, motivo por el que es importante conocer sus significados. Ekman y Friesen identificaron algunas de sus funciones más importantes que pasan por la ilustración, el reemplazo de la comunicación no verbal y la expresión de estados emocionales (Ekman y Friesen, 1967: 65).

Las manos funcionan como factores de ilustración acompañando a la palabra y reforzándola, por eso su utilización es constante en la docencia. Los ilustradores son movimientos que los docentes utilizan para hacerse entender así como para enfatizar. Son movimientos unidos al parlamento que aparecen con él de forma simultánea cuya función es la de acompanar lo que se dice verbalmente repitiendo, contradiciendo, acentuando o sustituyendo al mensaje oral. Son movimientos que pueden dibujar un pensamiento, señalar objetos, describir espacialmente o marcar el ritmo (Ekman y Friesen, 1967: 20). Estos ilustradores son habituales en la comunicación cara a cara.

Estos elementos kinésicos dependen de factores psicosomáticos individuales (como el carácter, el nerviosismo o la timidez); sociales, situacionales y culturales. Los ilustradores se dividen en seis subcategorías: bastones o batutas; ideógrafos, apuntadores, espaciales, pictógrafos, manos ventosa y kinetográficos (García Fernández, 2000: 49). La primera categoría, denominada ilustradores bastones o batuta, se utiliza para acentuar alguna parte concreta del discurso, para dar énfasis y suele marcar el ritmo del mensaje con las manos o los dedos. Los profesores los utilizan constantemente, de hecho sus manos apenas están quietas. La intensidad dependerá de la emoción que se imprima, por tanto el estado anímico será determinante. Ejemplos de estos gestos son el agarrón en el vacío, la mano apiñada, el índice alzado o la palma de la mano hacia arriba o hacia abajo. Mientras, los ilustradores ideógrafos son también muy comunes porque acompañan a la expresión de ideas discursivas o de tipo abstracto y dibujan una dirección del pensamiento, un itinerario lógico. Los gestos ideógrafos pueden referirse tanto a conceptos (frotarse los dedos de la mano para indicar algo sutil, esbozar garabatos en el aire para indicar complejidad) como a relaciones gramaticales entre oraciones (coordinación, yuxtaposición, adversatividad, etc.), acompañar a los adverbios de negación, afirmación o duda ('sí', 'no', 'quizá'), a los tiempos ('antes', después', 'mañana'), a los intensificadores ('mucho', 'algo', 'nada') y a los numerales ('primero', 'dos', etcétera).

Por otro lado, la descripción de un espacio que es nombrado verbalmente y que suele aludir al tamaño o la forma de algo recibe el nombre de ilustrador espacial. Estos gestos pueden ser estáticos (describen dimensiones) y dinámicos (se combinan con los deícticos: cuando un movimiento señalador es seguido de un 'barrido' del espacio con el índice). En cambio, los movimientos asociados a la siguiente categoría, los ilustradores pictógrafos, indican distancia entre la gente, los objetos o las ideas y 
ayudan a describir el aspecto formal del contenido verbal. Se utilizan tanto para enfatizar el mensaje (dibujar la silueta de un objeto) o para completarlo (dibujar un rectángulo para simular una caja). Asimismo, los ilustradores 'manos ventosa' son muy recomendables a la hora de argumentar una idea puesto que transmiten tranquilidad y aplomo. Este movimiento se escenifica al unir las yemas de los dedos de ambas manos mientras se emite el mensaje verbal. Es un gesto habitual entre los docentes. Finalmente, los ilustradores kinetográficos engloban los gestos de la mano o el brazo que acompañan a verbos y oraciones que describen movimientos simples o complejos.

Sin embargo, no son los ilustradores los únicos gestos que se realizan con las manos y los brazos. Los emblemas, "son actos no verbales que admiten una transposición oral directa o una definición de diccionario que consiste en una o dos palabras o en una frase" (Ekman, 1976: 14). Se trata del comportamiento no verbal más comprensible. Ejemplos de emblemas son el gesto que se utiliza en occidente para mostrar acuerdo -'ok'- y que se expresa bien con el dedo pulgar abierto apuntando hacia arriba y el resto de los dedos apretados, o también formando un anillo con el dedo pulgar e índice mientras se mantienen los demás extendidos; o el que simboliza la paz y que consiste en exhibir la mano con el dedo índice o corazón extendido formando una ' $\mathrm{V}$ '.

Pero quizá uno de los aspectos más interesantes para el objeto de este análisis reside en la expresión de estados emocionales a través de las manos. De hecho, esta circunstancia suele ser involuntaria (las personas nerviosas, cuando hablan en público, se agarran las manos con fuerza o juegan con objetos). Estos movimientos pueden revelar emociones específicas: muchos movimientos de las manos -como hurgarse las narices o rascarse- se relacionan con la autosatisfacción, tratándose de acciones que suelen reprimirse en público no siempre con éxito (rascarse o hurgarse las narices es un ataque a uno mismo, restregarse la cara aporta tranquilidad, etc.). Estos movimientos reciben el nombre de autoadaptadores y son conductas que se desarrollan en el transcurso de la niñez como esfuerzos de adaptación conscientes o inconscientes para satisfacer necesidades, dominar emociones y desarrollar contactos, así como para superar la tensión.

\section{Hacia un inVENTARio de los gestos manuales más Recurrentes EN EL PROFESORADO UNIVERSITARIO}

Dedicaremos este apartado a realizar una síntesis de algunos de los gestos de las manos más comunes que muestran los docentes hacia sus alumnos.

En primer lugar destacamos la acción de frotarse las manos, un gesto con el que el individuo indica que espera algo bueno ya que comunica 
expectativas positivas (Pease, 2006: 144-157). Otro de los gestos destacable es el de las manos unidas, una posición que muestra una actitud reprimida, ansiosa o negativa. También es habitual en el repertorio del docente el denominado 'campanario'. Para realizarlo, los dedos de la mano presionan los de la otra hasta formar una especie de campanario que a veces se balancea. Indica confianza y seguridad, por lo que es recomendable al impartir clase. Sin embargo, debería evitarse cuando alguien intenta ser convincente ya que puede interpretarse en clave de arrogancia (sobre todo si el gesto aparece junto a una inclinación hacia atrás de la cabeza).

Uno de los gestos asociados a la superioridad, la confianza y el poder es el de las manos unidas a la espalda. De la misma forma, cuando alguien enseña los pulgares, al ser los dedos más fuertes, indica superioridad, un motivo por el que se utilizan para transmitir dominio o actitudes agresivas. Otras veces los pulgares sobresalen por los bolsillos como si el individuo intentase esconder su dominio. Asimismo, los brazos cruzados con los pulgares hacia arriba es una señal que indica actitud defensiva y superioridad.

Pero además de los citados gestos, a la hora de explicar conceptos en el aula es importante ser consciente de los diferentes significados que puede transmitir la palma de la mano en función de su posición. Mostrar la mano ha sido a lo largo de la historia símbolo de sinceridad, honestidad, vasallaje y sumisión. Una de las pistas para detectar si alguien es abierto o sincero consiste en observar la exhibición de las palmas de sus manos ya que el ser humano las utilizaba para mostrar que iba desarmado, es decir, que no era fuente de amenaza. Cuando alguien empieza a abrirse o a ser sincero expone toda la palma de su mano o parte de ella. Es un gesto inconsciente que se interpreta en términos de franqueza. De la misma forma, un individuo que quiere que no le descubran en algún renuncio suele esconder sus manos o cruzarse de brazos (Pease, 2006: 47-52).

Una de las señales más poderosas es la que transmite la mano cuando da instrucciones u órdenes. Existen tres gestos de orden principales realizados con la palma de la mano: hacia arriba, hacia abajo y cerrada. La palma de la mano hacia arriba se interpreta como un gesto de sumisión y está libre de amenazas. Se asocia con la honestidad. Es un gesto de transparencia y deferencia (Mínguez Vela, 1999: 64). En este sentido cabe destacar que la presentadora estadounidense Oprah Winfrey, quien ha conducido el magazín de variedades más visto del mundo, muestra un gran apertura a la comunicación a través de las palmas de sus manos. A pesar de ser una persona que comunica poco lo que siente, está totalmente orientada hacia los demás. Tiene los antebrazos muy móviles y las palmas de su mano se dirigen al público cuando está en la sala durante más del $60 \%$ del tiempo. Al mostrar las palmas, establece de forma inconsciente un vínculo entre el público de la sala, los telespectadores y los invitados. Una de las razones de la popularidad de Winfrey es su forma de avanzar con las pal- 
mas abiertas, con una transparencia total. Así establece un potente vínculo entre quienes participan en la emisión y quienes la siguen en casa (Turchet, 2005: 140). Sin embargo, la palma hacia abajo proyecta autoridad inmediata. Cuando alguien da órdenes con la mano hacia abajo, el que las recibe puede experimentar sentimientos antagónicos. Lo realizan personas de estatus superior al interlocutor. De hecho, el saludo nazi se realizaba con la palma de la mano mirando hacia abajo, siendo el símbolo de la tiranía. Finalmente, la posición de la palma de la mano cerrada con un dedo apuntando se utiliza cuando la persona que habla golpea figuradamente a quien le escucha como signo de sumisión. Es un gesto agresivo que evoca sentimientos negativos y suele molestar.

Este recorrido por los movimientos de manos no puede concluir sin mencionar el denominado gesto 'del anillo'. Para realizarlo es preciso unir el anular y el índice formando un círculo y dejar estirados el resto de dedos apuntando hacia arriba. Su significado es común en todos los países de habla inglesa aunque desde allí se ha exportado gracias a la televisión. Es interesante resaltar que cuando un docente muestra el gesto del anillo ofrece una imagen autoritaria pero no agresiva. Quien lo exhibe parece reflexivo, objetivo y centrado (Pease, 2006: 137).

\section{MATERIAL y MÉTODOS}

Con el objetivo de comprobar que los profesores más hábiles a la hora de desplegar su repertorio de gestos manuales tienen menos dificultades para transmitir conocimientos a sus alumnos, la investigación ha empleado una metodología de análisis basada en dos técnicas diferentes. Por un lado, a la hora de diseccionar los movimientos de manos más recurrentes en los docentes, se ha utilizado el análisis de contenido, una técnica idónea para el estudio del lenguaje kinésico desplegado en el aula debido a las posibilidades que ofrece para conocer la estructura del mensaje no verbal. Sin embargo, este estudio va más allá y para comprobar la percepción que los alumnos tienen de sus profesores y las actitudes que proyectan, se han realizado una serie de encuestas que completan y refuerzan el análisis ya que permiten comparar los resultados de ambos métodos.

\subsection{El análisis de contenido aplicado a los movimientos manuales}

Una de las técnicas de investigación más eficaz para el análisis del comportamiento kinésico (movimientos de manos) del profesorado universitario es el análisis de contenido cuantitativo, puesto que posibilita la categorización de aquellos símbolos no verbales más representativos en 
función de sus actitudes y estados de ánimo. Este análisis permite aplicar sistemáticamente unas reglas que miden la frecuencia con que aparecen unos elementos de interés en el conjunto de una masa de información seleccionada para estudiar algunos de los aspectos que parecen más útiles de acuerdo a los propósitos del trabajo. Además, facilita la tarea de procesar gran volumen de información con fiabilidad (Berganza y Ruiz San Román, 2005: 213).

Así, para poder efectuar el análisis se diseñó una ficha de registro que permitió realizar el análisis estadístico. Esta ficha es un extracto de la ficha matriz diseñada en el artículo 'Investigar en comunicación no verbal: un modelo para el análisis del comportamiento kinésico de líderes políticos y para la determinación de su significación estratégica' (Hernández Herrarte y Rodríguez Escanciano, 2009: 61-94), aplicada en varias investigaciones previamente con resultados significativos sobre el valor comunicativo de distintos personajes. En esta ficha se presenta un conjunto de categorías kinésicas entre las que destacan diferentes movimientos realizados con las manos, una de las partes del cuerpo donde se apoya la mayor parte de la expresividad discursiva de los docentes universitarios.

Hemos tomado como muestra de análisis cuatro clases magistrales de 50 minutos de duración cada una impartidas por cuatro profesores diferentes (que en el trabajo se han denominado Prof. 1; Prof. 2; Prof. 3 y Prof. 4 con el objetivo de preservar su anonimato), todos ellos titulares de las cuatro asignaturas del segundo semestre pertenecientes al $5^{\circ}$ curso de la licenciatura en Publicidad y Relaciones Públicas de la Universidad Europea Miguel de Cervantes. Los datos fueron obtenidos mediante la grabación de las clases. Para ello, se dispuso de una cámara de vídeo que grabó la clase completa de forma automática, sin necesidad de operador puesto que así no se condicionó el desarrollo de la docencia. Todas las grabaciones se realizaron en clases teóricas en las que se explicó a los alumnos conceptos relacionados con las materias y se llevaron a cabo durante el mes de mayo de 2013.

Por lo que respecta a la categorización, el inventario gestual de las manos se ha desglosado en varias categorías y subcategorías que se presentan a continuación en la ficha de análisis. Para una mayor comprensión de los gestos, el significado de los movimientos aparece junto a cada categoría (ver Tabla 1).

El análisis del comportamiento kinésico observado se llevó a cabo efectuando un estudio estadístico de los datos obtenidos. Para la codificación se eligió un sistema binario de presencia-ausencia en el que los valores numéricos $(1,2,3$, etc.) representan la presencia y el 0 la ausencia. La cuantificación se basa en la estadística descriptiva univariada (frecuencias y porcentajes). Así, el número de frecuencias en porcentajes se efectuó 
observando el número de veces que aparece repetido un mismo valor de una variable. Tras determinarlo, se trabajó con tantos por ciento, dado que facilita la comparación de los resultados y la redacción de las conclusiones. Este análisis se realizó con el programa informático Excel, una aplicación que permite representar gráficamente los resultados facilitando su interpretación.

Para poder comprobar si ciertos movimientos de manos se asocian a actitudes positivas que facilitan el aprendizaje, el análisis se completó con una serie de encuestas realizadas a todos los alumnos matriculados en las clases objeto de estudio. La encuesta fue realizada por 13 alumnos de forma anónima. Los resultados de estas encuestas permiten comparar los resultados y determinar hasta qué punto el lenguaje de las manos del docente influye en la asimilación de conceptos tomando como referencia el punto de vista del receptor. La encuesta que se pasó a los alumnos aparece en el anexo $\mathrm{n}^{\mathrm{o}} 1$.

TABLA 1. Ficha de análisis de contenido de la CNV

\begin{tabular}{|c|c|c|}
\hline & $\begin{array}{c}\text { SIGNIFICADO } \\
\text { CONCEPTUAL } \\
\text { DEL ELEMENTO }\end{array}$ & $\begin{array}{l}\text { SIGNIFICADO } \\
\text { PERSUASIVO } \\
\text { APLICADO }\end{array}$ \\
\hline \multicolumn{3}{|l|}{ BRAZOS } \\
\hline Brazos abajo & & $\begin{array}{l}\text { Posición neutral. Pero } \\
\text { si aparecen desligados } \\
\text { del propio cuerpo } \\
\text { puede transmitir } \\
\text { falta de seguridad } \\
\text { y credibilidad } \\
\text { NEGATIVO O NEUTRO }\end{array}$ \\
\hline Brazos arriba & & $\begin{array}{l}\text { Saludo, triunfo. Se logra } \\
\text { mayor visibilidad. } \\
\text { Vitalidad } \\
\text { POSITIVo }\end{array}$ \\
\hline Brazos abiertos & & $\begin{array}{l}\text { Gesto que invita } \\
\text { al abrazo, petición } \\
\text { de aplauso y apertura } \\
\text { a la comunicación } \\
\text { POSITIVo }\end{array}$ \\
\hline $\begin{array}{l}\text { Brazos abajo } \\
\text { con puños cerrados }\end{array}$ & & $\begin{array}{l}\text { Cierta tensión } \\
\text { y nerviosismo } \\
\text { NEGATIVO }\end{array}$ \\
\hline
\end{tabular}


TABla 1. (cont.)

\begin{tabular}{|c|c|c|}
\hline & $\begin{array}{c}\text { SIGNIFICADO } \\
\text { CONCEPTUAL } \\
\text { DEL ELEMENTO }\end{array}$ & $\begin{array}{c}\text { SIGNIFICADO } \\
\text { PERSUASIVO } \\
\text { APLICADO }\end{array}$ \\
\hline \multicolumn{3}{|l|}{ BRAZOS (cont.) } \\
\hline $\begin{array}{l}\text { Brazos hacia delante } \\
\text { con manos que empujan }\end{array}$ & & Rechazo \\
\hline $\begin{array}{l}\text { Brazos hacia delante } \\
\text { con puños cerrados }\end{array}$ & & Agresión \\
\hline $\begin{array}{l}\text { Uno o ambos brazos } \\
\text { cruzados sobre el pecho }\end{array}$ & & $\begin{array}{l}\text { Se identifica } \\
\text { con actitudes defensivas } \\
\text { NEGATIVO }\end{array}$ \\
\hline $\begin{array}{l}\text { Cruce de brazos } \\
\text { reforzado }\end{array}$ & Incluye los puños cerrados & $\begin{array}{l}\text { Hostilidad y postura } \\
\text { defensiva } \\
\text { NEGATIVO }\end{array}$ \\
\hline Brazos en jarras & $\begin{array}{l}\text { Los brazos se colocan } \\
\text { a la altura de las caderas }\end{array}$ & $\begin{array}{l}\text { Es una situación } \\
\text { defensiva ante una } \\
\text { amenaza } \\
\text { NEGATIVo }\end{array}$ \\
\hline $\begin{array}{l}\text { El abrazo } \\
\text { de los dos brazos }\end{array}$ & $\begin{array}{l}\text { Las manos de la persona } \\
\text { rodean con fuerza } \\
\text { los antebrazos para evitar } \\
\text { la exposición de la parte } \\
\text { frontal del cuerpo. }\end{array}$ & \begin{tabular}{l} 
Es una actitud \\
reprimida. \\
Provoca rechazo. \\
Bloquea la interacción \\
con el receptor. \\
\multicolumn{1}{c}{ NEGATIVO }
\end{tabular} \\
\hline $\begin{array}{l}\text { Cruce de brazos } \\
\text { con pulgares hacia arriba }\end{array}$ & & $\begin{array}{l}\text { Orgullo de sí mismo } \\
\text { y control de la situación. } \\
\text { POSITIVo }\end{array}$ \\
\hline Cruce de brazos simétrico & & $\begin{array}{l}\text { Posición defensiva } \\
\text { pero sumisa } \\
\text { NEGATIVO }\end{array}$ \\
\hline $\begin{array}{l}\text { Estrechar la mano } \\
\text { con uno mismo }\end{array}$ & $\begin{array}{l}\text { Se cruzan los brazos } \\
\text { a la altura de los genitales. }\end{array}$ & $\begin{array}{r}\text { Derrota y vulnerabilidad } \\
\text { NEGATIVO }\end{array}$ \\
\hline $\begin{array}{l}\text { Formas camufladas } \\
\text { de cruzar los brazos }\end{array}$ & $\begin{array}{l}\text { Los brazos sujetan bolsos, } \\
\text { pulseras, relojes o gemelos, } \\
\text { etcétera. }\end{array}$ & $\begin{array}{l}\text { Nerviosismo, ansiedad. } \\
\text { NEGATIVO }\end{array}$ \\
\hline Frotar las manos & & $\begin{array}{r}\text { Ansiedad. Autoritarismo } \\
\text { NEGATIVO }\end{array}$ \\
\hline
\end{tabular}


TABLA 1. (cont.)

\begin{tabular}{|c|c|c|}
\hline & $\begin{array}{c}\text { SIGNIFICADO } \\
\text { CONCEPTUAL } \\
\text { DEL ELEMENTO }\end{array}$ & $\begin{array}{l}\text { SIGNIFICADO } \\
\text { PERSUASIVO } \\
\text { APLICADO }\end{array}$ \\
\hline \multicolumn{3}{|l|}{ MANOS } \\
\hline $\begin{array}{l}\text { Palma de la mano } \\
\text { hacia arriba }\end{array}$ & & $\begin{array}{l}\text { Transparencia, } \\
\text { honestidad. } \\
\text { Capacidad de diálogo. } \\
\text { POSITIVo }\end{array}$ \\
\hline $\begin{array}{l}\text { Palma de la mano } \\
\text { hacia abajo }\end{array}$ & & $\begin{array}{l}\text { Autoridad inmediata. } \\
\text { Tiranía. Escasa } \\
\text { capacidad de diálogo. } \\
\text { NEGATIVO }\end{array}$ \\
\hline Puño cerrado levantado & & $\begin{array}{l}\text { Amenaza, agresividad } \\
\text { y enfado } \\
\text { NEGATIVO }\end{array}$ \\
\hline Ambos puños cerrados & & $\begin{array}{r}\text { Agresividad y hostilidad } \\
\text { NEGATIVO }\end{array}$ \\
\hline Manos unidas & & $\begin{array}{l}\text { Muestra una actitud } \\
\text { reprimida, ansiosa } \\
\text { o negativa. } \\
\text { NEGATIVO }\end{array}$ \\
\hline El campanario & $\begin{array}{l}\text { Los dedos de una mano } \\
\text { presionan ligeramente } \\
\text { los de la otra formando } \\
\text { un campanario }\end{array}$ & 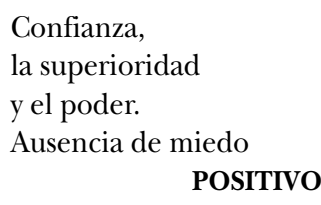 \\
\hline Manos unidas a la espalda & & $\begin{array}{l}\text { Superioridad, confianza } \\
\text { y poder } \\
\text { POSITIVO }\end{array}$ \\
\hline Mano que sujeta muñeca & & $\begin{array}{l}\text { Frustración e intento } \\
\text { de autocontrol } \\
\text { NEGATIVO }\end{array}$ \\
\hline Mano que sujeta el brazo & & $\begin{array}{l}\text { Enojo y frustración } \\
\text { NEGATIVO }\end{array}$ \\
\hline
\end{tabular}


TABLA 1. (cont.)

\begin{tabular}{|c|c|c|}
\hline & $\begin{array}{c}\text { SIGNIFICADO } \\
\text { CONCEPTUAL } \\
\text { DEL ELEMENTO }\end{array}$ & $\begin{array}{l}\text { SIGNIFICADO } \\
\text { PERSUASIVO } \\
\text { APLICADO }\end{array}$ \\
\hline \multicolumn{3}{|l|}{ MANOS (cont.) } \\
\hline Enseñar los pulgares & & $\begin{array}{l}\text { Dominio, aseveración } \\
\text { o actitudes agresivas } \\
\text { NEGATIVO }\end{array}$ \\
\hline El gesto del anillo & $\begin{array}{l}\text { Para realizar este gesto } \\
\text { es preciso unir el anular } \\
\text { y el índice formando } \\
\text { un círculo y dejar estirados } \\
\text { el resto de dedos } \\
\text { apuntando hacia arriba }\end{array}$ & $\begin{array}{l}\text { Los oradores } \\
\text { que mientras hablan } \\
\text { muestran el gesto } \\
\text { de 'Ok' suelen ofrecer } \\
\text { una imagen autoritaria } \\
\text { pero no agresiva. Actitud } \\
\text { reflexiva y centrada } \\
\text { NEGATIVo }\end{array}$ \\
\hline $\begin{array}{l}\text { Autoadaptadores } \\
\text { (sujetar objetos: bolis, } \\
\text { papeles, etcétera) }\end{array}$ & & $\begin{array}{l}\text { Satisfacen necesidades } \\
\text { de tipo somático. } \\
\text { Palían el nerviosismo } \\
\text { POSITIVo }\end{array}$ \\
\hline $\begin{array}{l}\text { Autoadaptadores } \\
\text { (rascarse, mesarse el pelo, } \\
\text { etcétera) }\end{array}$ & & $\begin{array}{l}\text { Satisfacen necesidades } \\
\text { de tipo somático. En este } \\
\text { caso denota nerviosismo, } \\
\text { tensión y falta de control } \\
\text { de la situación. } \\
\text { NEGATIVo }\end{array}$ \\
\hline $\begin{array}{l}\text { Autoadaptadores } \\
\text { (utilizar la pizarra) }\end{array}$ & & $\begin{array}{l}\text { Ayuda a clarificar } \\
\text { la explicación } \\
\text { y a asentar conceptos. } \\
\text { NEUTRO }\end{array}$ \\
\hline $\begin{array}{l}\text { GESTOS CON MANOS } \\
\text { ILUSTRADORES }\end{array}$ & $\begin{array}{l}\text { Son aquellos movimientos } \\
\text { que se emplean } \\
\text { para hacerse entender } \\
\text { y para enfatizar ciertas } \\
\text { partes del discurso. }\end{array}$ & \\
\hline $\begin{array}{l}\text { Ilustradores } \\
\text { bastones/batuta }\end{array}$ & $\begin{array}{l}\text { Suelen marcar el ritmo } \\
\text { del mensaje con las manos }\end{array}$ & $\begin{array}{l}\text { Se utilizan para remarcar } \\
\text { alguna parte concreta } \\
\text { del discurso, para dar } \\
\text { énfasis. } \\
\text { POSITIVo }\end{array}$ \\
\hline
\end{tabular}


TABLA 1. (cont.)

\begin{tabular}{|c|c|c|}
\hline & $\begin{array}{c}\text { SIGNIFICADO } \\
\text { CONCEPTUAL } \\
\text { DEL ELEMENTO }\end{array}$ & $\begin{array}{l}\text { SIGNIFICADO } \\
\text { PERSUASIVO } \\
\text { APLICADO }\end{array}$ \\
\hline \multicolumn{3}{|l|}{$\begin{array}{c}\text { GESTOS CON MANOS } \\
\text { ILUSTRADORES } \\
(\text { cont. })\end{array}$} \\
\hline Ilustradores ideógrafos & $\begin{array}{l}\text { Dibujan una dirección } \\
\text { del pensamiento. }\end{array}$ & $\begin{array}{l}\text { Suelen acompañar } \\
\text { a la expresión de ideas } \\
\text { discursivas o de tipo } \\
\text { abstracto } \\
\text { NEUTRO }\end{array}$ \\
\hline Ilustradores apuntadores & $\begin{array}{l}\text { Son movimientos deícticos } \\
\text { que muestran algo } \\
\text { dirigiéndose a algo o a } \\
\text { alguien. Sirven por tanto } \\
\text { para señalar la situación } \\
\text { espacial o para identificar } \\
\text { cosas. }\end{array}$ & $\begin{array}{l}\text { Pueden resultar } \\
\text { amenazantes } \\
\text { NEGATIVO O NEUTRO }\end{array}$ \\
\hline Ilustradores espaciales & $\begin{array}{l}\text { Describen un espacio } \\
\text { que es nombrado } \\
\text { verbalmente: tamaños } \\
\text { o formas. }\end{array}$ & $\begin{array}{l}\text { Ayudan a ilustrar } \\
\text { el discurso verbal } \\
\text { POSITIVO }\end{array}$ \\
\hline Ilustradores pictógrafos & $\begin{array}{l}\text { El movimiento indica } \\
\text { distancia entre la gente, } \\
\text { los objetos o las ideas }\end{array}$ & $\begin{array}{l}\text { Ayudan a describir } \\
\text { el aspecto formal } \\
\text { del contenido verbal. } \\
\text { Se utilizan tanto para } \\
\text { enfatizar el mensaje oral } \\
\text { (dibujar la silueta } \\
\text { de un objeto) o para } \\
\text { completarlo (dibujar } \\
\text { un rectángulo para } \\
\text { simbolizar una caja) } \\
\text { POSITIVO }\end{array}$ \\
\hline $\begin{array}{l}\text { Ilustradores } \\
\text { manos ventosa }\end{array}$ & $\begin{array}{l}\text { Se unen las yemas de los } \\
\text { dedos de ambas manos }\end{array}$ & $\begin{array}{l}\text { Tranquilidad, aplomo } \\
\text { y seguridad. } \\
\text { POSITIVo }\end{array}$ \\
\hline $\begin{array}{l}\text { Ilustradores } \\
\text { kinetográficos }\end{array}$ & $\begin{array}{l}\text { Acompañan a verbos } \\
\text { y oraciones que describen } \\
\text { movimientos simples }\end{array}$ & $\begin{array}{l}\text { Acompañan a los verbos } \\
\text { y a las oraciones que } \\
\text { describen movimientos. } \\
\text { POSITIVO }\end{array}$ \\
\hline
\end{tabular}




\section{ANÁlisis y RESULTADOS}

A continuación se ofrecen los principales resultados obtenidos fruto del análisis de contenido basado en los movimientos de manos de los cuatro docentes observados:

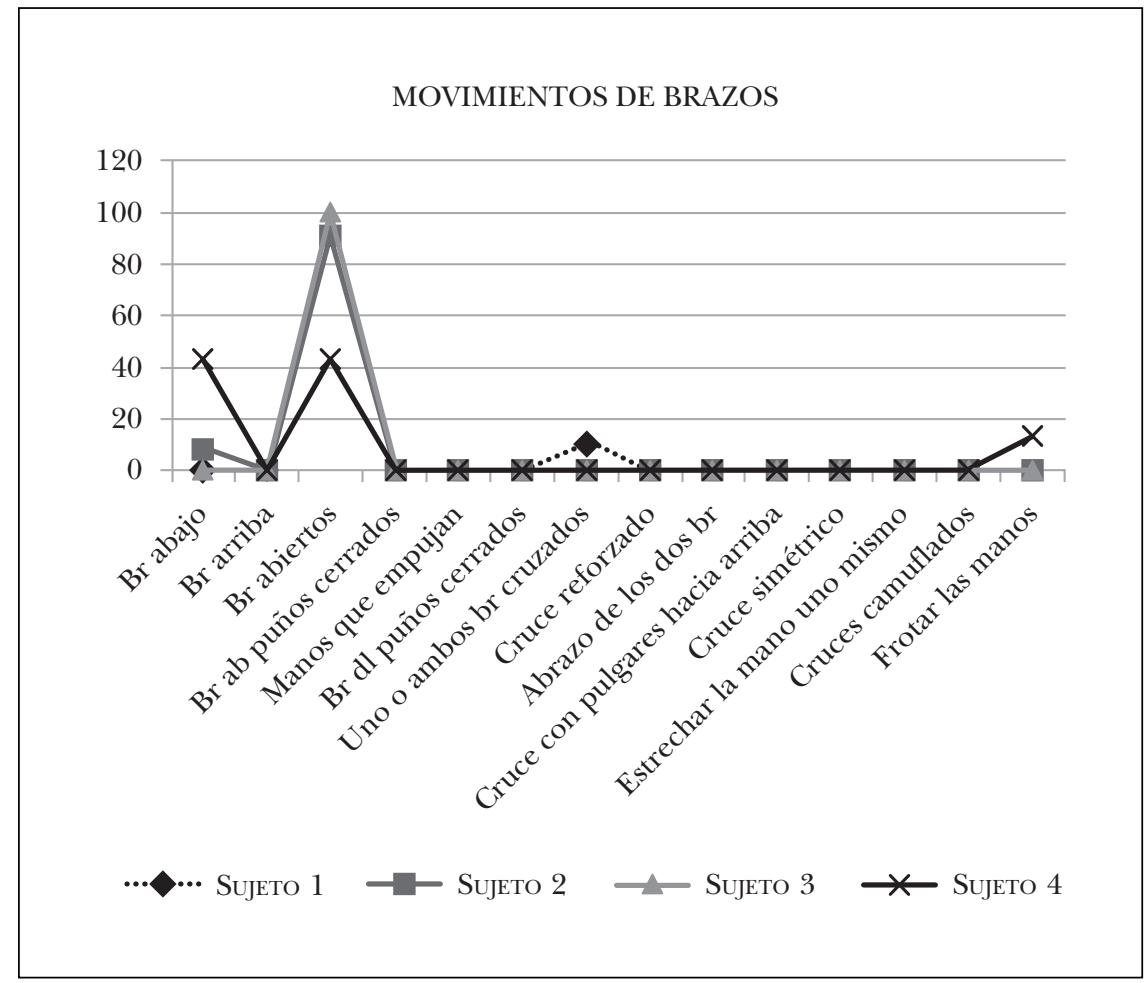

En cuanto a los movimientos de brazos, la gráfica es muy clarificadora al mostrar escasos y poco variados gestos de este tipo. El movimiento que destaca sobre todos los demás es el de brazos abiertos'. De hecho, el sujeto 1 lo mostró un $90 \%$ del tiempo; el sujeto 2 un $90,91 \%$; el sujeto $3 \mathrm{du}$ rante la totalidad del tiempo observado y el sujeto 4 un 43,48\%. Este gesto se interpreta en clave de positividad, puesto que se relaciona con la empatía y la apertura a la comunicación. Los brazos abajo fue otra de las posturas observadas. En el caso del sujeto 1 en el 9,09\% del tiempo observado y el $43,4 \%$ por parte del sujeto 4 (este gesto refleja cansancio y dejadez). Asimismo, el sujeto 1 mantuvo los brazos cruzados un $10 \%$ del tiempo, un aspecto negativo ya que se identifica con actitudes defensivas siendo una 
barrera comunicativa. Finalmente, añadir que el sujeto 4 se frotó las manos un $13,04 \%$ del tiempo, algo que no le beneficia puesto que refleja ambición y ansiedad.

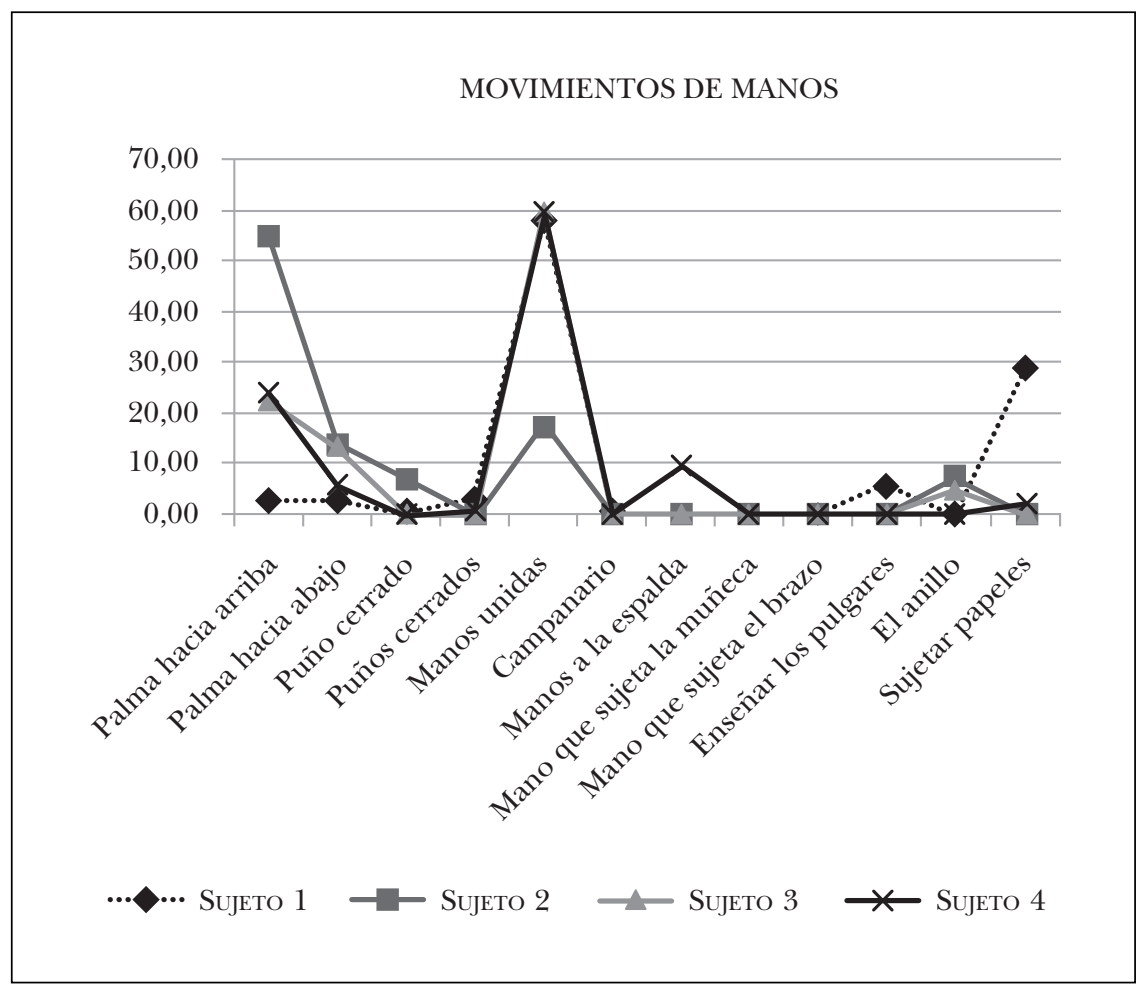

Las manos de los profesores objeto de análisis presentaron mayor abanico de movimientos. Detallamos los más recurrentes: el gesto que domina en la gráfica es sin duda el de las manos unidas, que pudo verse en un $57,8 \%$ del tiempo en el caso del sujeto 1 ; un $17,2 \%$ en el sujeto 2; un $59 \%$ en el sujeto 3 y un $59,2 \%$ en el caso del sujeto 4 . Es un gesto que interpretamos como poco conveniente puesto que denota represión y frustración. Por otro lado, las palmas de las manos hacia arriba pudieron verse un $2,63 \%$ del tiempo en el sujeto 1 ; un $55,17 \%$ en el 2 ; un $22,73 \%$ en el 3 y un $24,97 \%$ en el 4 . Se trata de un gesto que garantiza el éxito comunicativo al asociarse a la transparencia, la sinceridad y el no tener nada que ocultar. Sin embargo, la gráfica destaca también la recurrencia de las palmas hacia abajo (un 2,63\% del tiempo en el sujeto 1; un $13,79 \%$ en el 2; un $13,64 \%$ en el 3 y un $5,56 \%$ en el 4 ). Este gesto es de los que hay que inten- 
tar evitar puesto que transmite autoritarismo y agresividad. Es interesante comentar también que el sujeto 1 mostró los puños cerrados el 2,63\% del tiempo (es un gesto agresivo y autoritario); el sujeto 2 hizo lo mismo con un solo puño el 6,9\% del tiempo (también se asocia a la agresividad no controlada); el sujeto 4 estuvo un 9,26\% del tiempo con las manos en la espalda (superioridad, confianza y poder); el sujeto 1 enseñó los pulgares un $5,26 \%$ del tiempo (dominio y agresividad); los sujetos 2 y 3 exhibieron el gesto de anillo, que se relaciona con actitudes centradas y reflexivas (un $6,9 \%$ y un $4,5 \%$ del tiempo respectivamente) y, finalmente, la sujeción de papeles fue un recurso utilizado un $28,95 \%$ del tiempo por parte del sujeto 1 y un $1,85 \%$ en el caso del 2. En este contexto es un gesto neutro que se interpreta como búsqueda y apoyo a la información que se está transmitiendo en el aula.

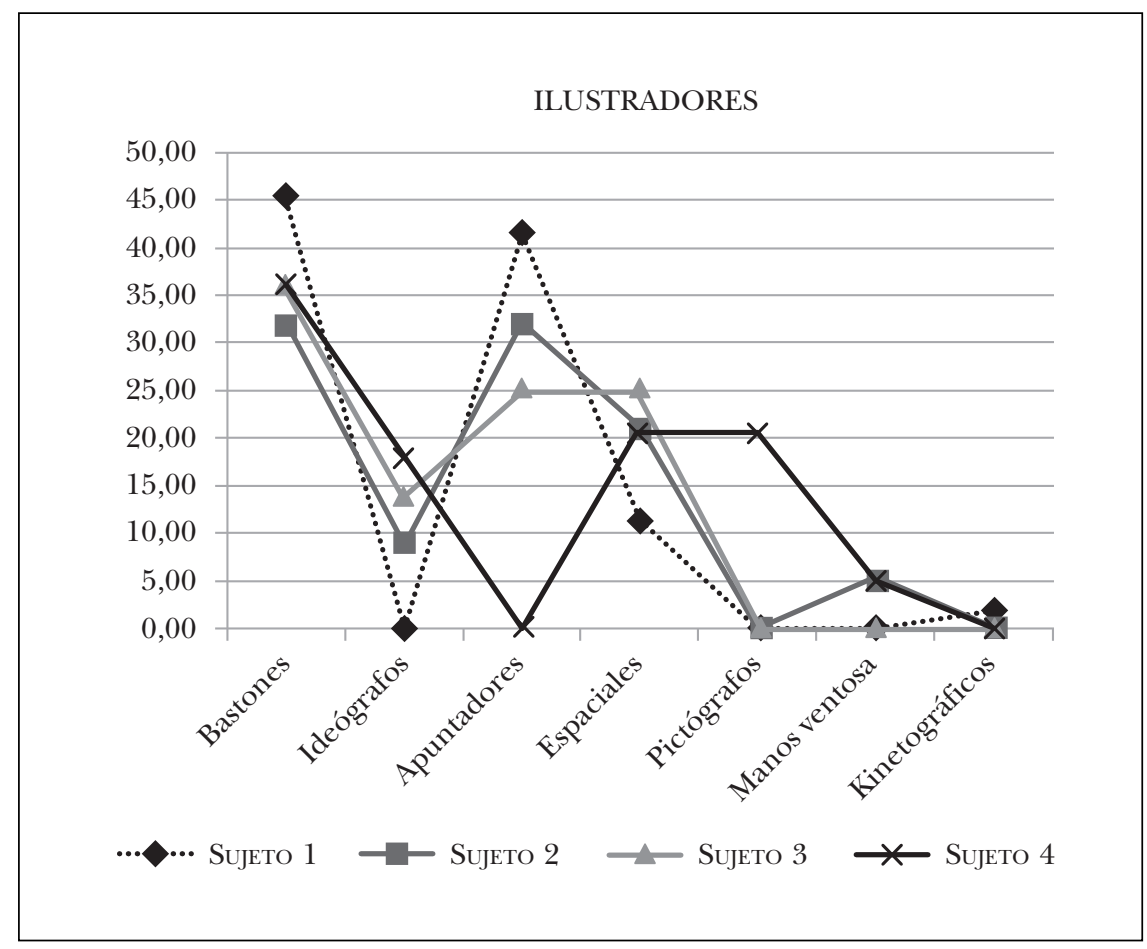

Los ilustradores son los movimientos que mayor heterogeneidad presentan. Son los denominados 'bastones' los más repetidos (un 45,2\% del tiempo en el caso del sujeto 1 ; un $32,1 \%$ en el caso del 2; un $35,9 \%$ en el caso del 3 y un $35,9 \%$ en el caso del 4 ). Son movimientos que remarcan 
partes del discurso y aportan énfasis, siendo muy deseables en el contexto del aula. Asimismo, también fueron muy redundantes los 'apuntadores' (45,1\% del tiempo en el sujeto $1 ; 32,1 \%$ en el $2 ; 25 \%$ en el 3 y $0 \%$ en el 4$)$. Estos movimientos señalan y centran la atención en algún aspecto concreto del discurso, por lo que también son aceptables. De la misma forma, los ilustradores 'espaciales' que describen el tamaño y forma de las cosas ayudando a ilustrar el discurso pudieron verse un 11,3\% del tiempo en el sujeto 1 ; un $21,4 \%$ en el caso del 2; un $25 \%$ en el 3 y un $20,5 \%$ en el 4 . Otros movimientos observados con menor intensidad fueron los ilustradores 'ideógrafos' que ayudan a materializar ideas abstractas $(0 \%$ en el caso del sujeto $1 ; 8,93 \%$ en el $2 ; 14,6 \%$ en el 3 y $17,9 \%$ en el caso del 4 . Asimismo, los ilustradores 'pictógrafos', que enfatizan y completan el mensaje, pudieron verse un 20,5\% del tiempo en la disertación del sujeto 4 . Por su parte, las 'manos ventosa' (asociadas a la tranquilidad, el aplomo y la seguridad, se observaron solo en el sujeto 2 y 3 en un 5,3 y 5,1\% del tiempo observado respectivamente. Finalmente, el sujeto 1 exhibió tan solo en un 1,8\% del tiempo ilustradores 'kinetográficos' (acompañan a los verbos y oraciones describiendo movimientos).

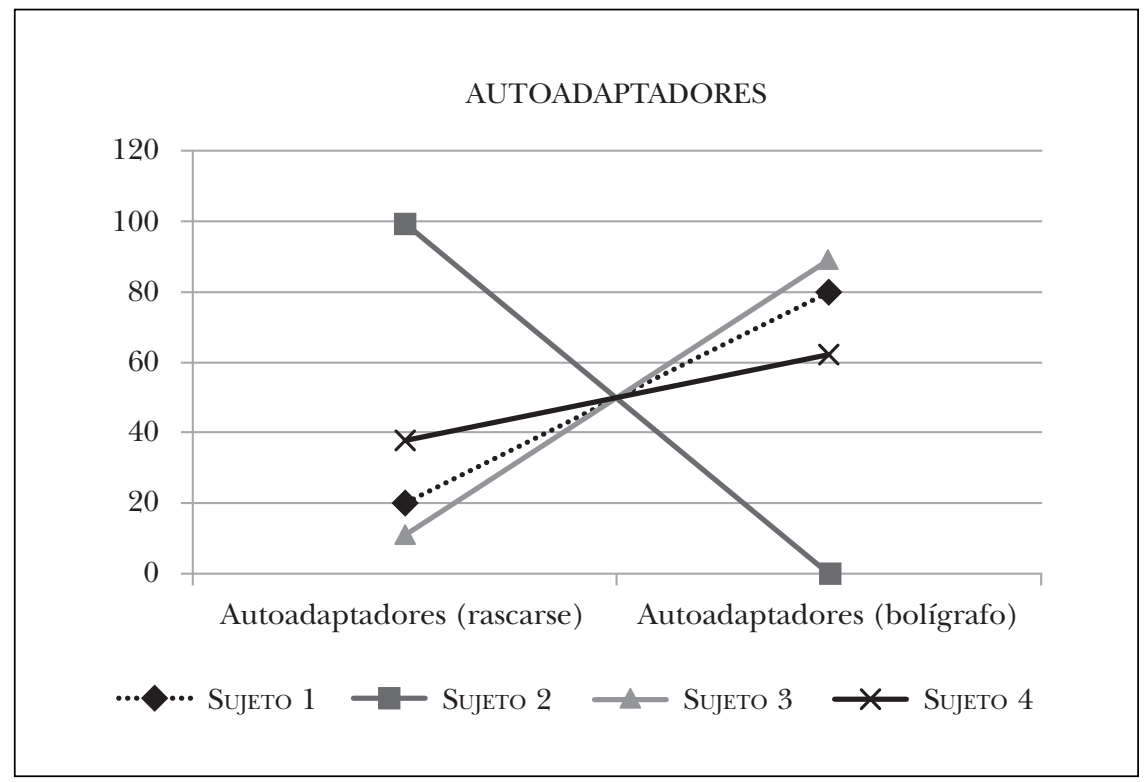

Tal y como muestra el gráfico, el sujeto 2 se toca el pelo y se frota el rostro mientras imparte docencia, un gesto relacionado con la tensión. Mientras, los sujetos 1, 3 y 4 agarran un bolígrafo cuando disertan, un auto- 
adaptador que ayuda a calmar los nervios cuando no se sabe muy bien qué hacer con las manos. El sujeto 1 utilizó este recurso en un $80 \%$ del tiempo observado, el sujeto 3 en un $88,8 \%$ del tiempo y el 4 en un $61,90 \%$.

A continuación se detallan los resultados del procesamiento de los datos rescatados de las encuestas realizadas por los alumnos participantes en el estudio:

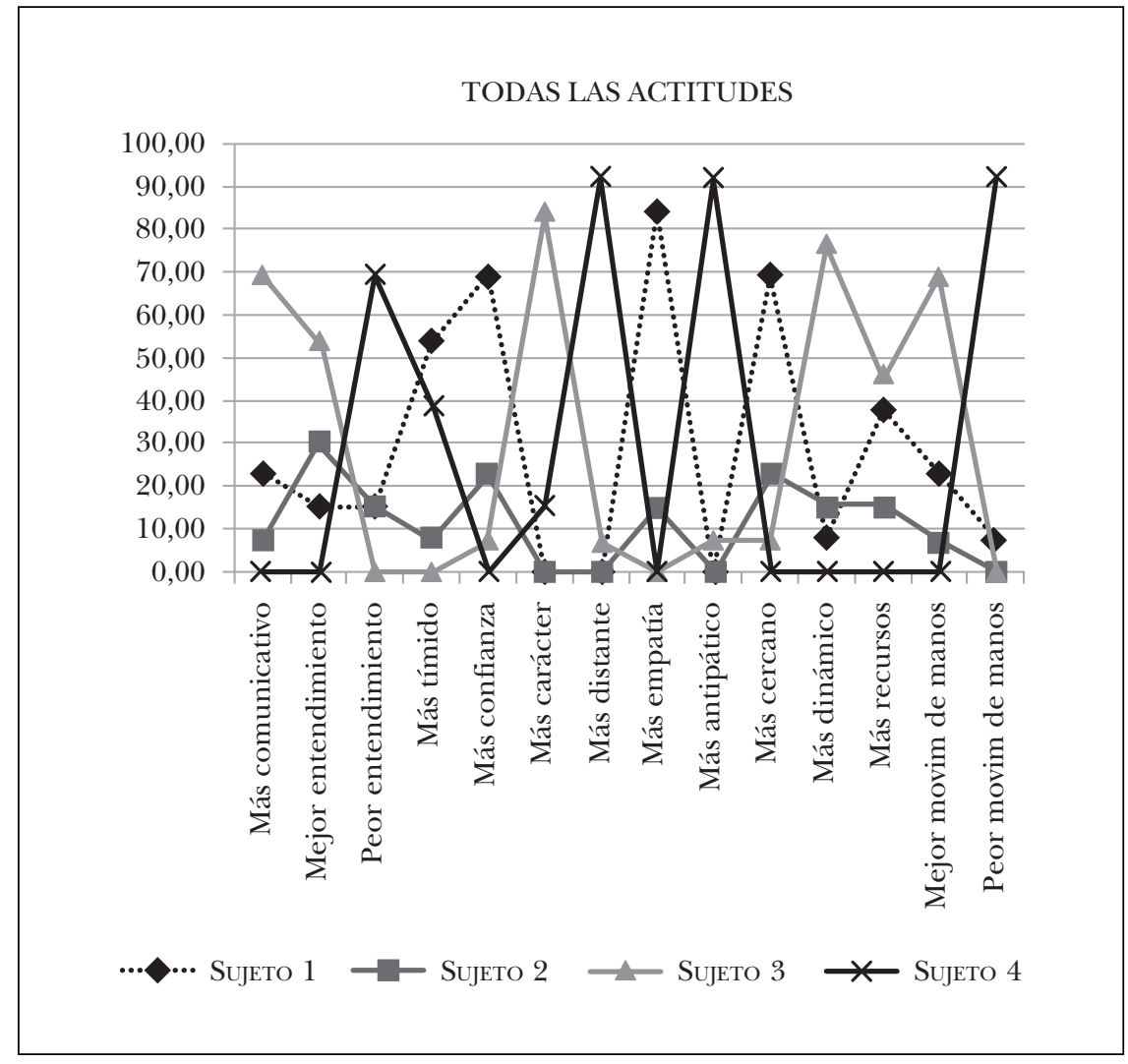

Los alumnos que cursan $5^{\circ}$ de Publicidad califican al sujeto 1 y 3 como los docentes con actitudes no verbales más positivas, un aspecto que repercute sin duda en el éxito comunicativo de ambos al impartir sus clases teóricas. Con más detalle: el sujeto 3 aparece como el más comunicativo (para el 69,2\% de los encuestados), siendo además el que mejor se hace entender (para el 53,8\% de los alumnos), el más dinámico $(76,9 \%$ ), el que aporta más recursos $(46,1 \%)$ y el que les parece que maneja mejor las manos en el aula $(69,2 \%)$. Mientras, el sujeto 1 es el considerado más con- 


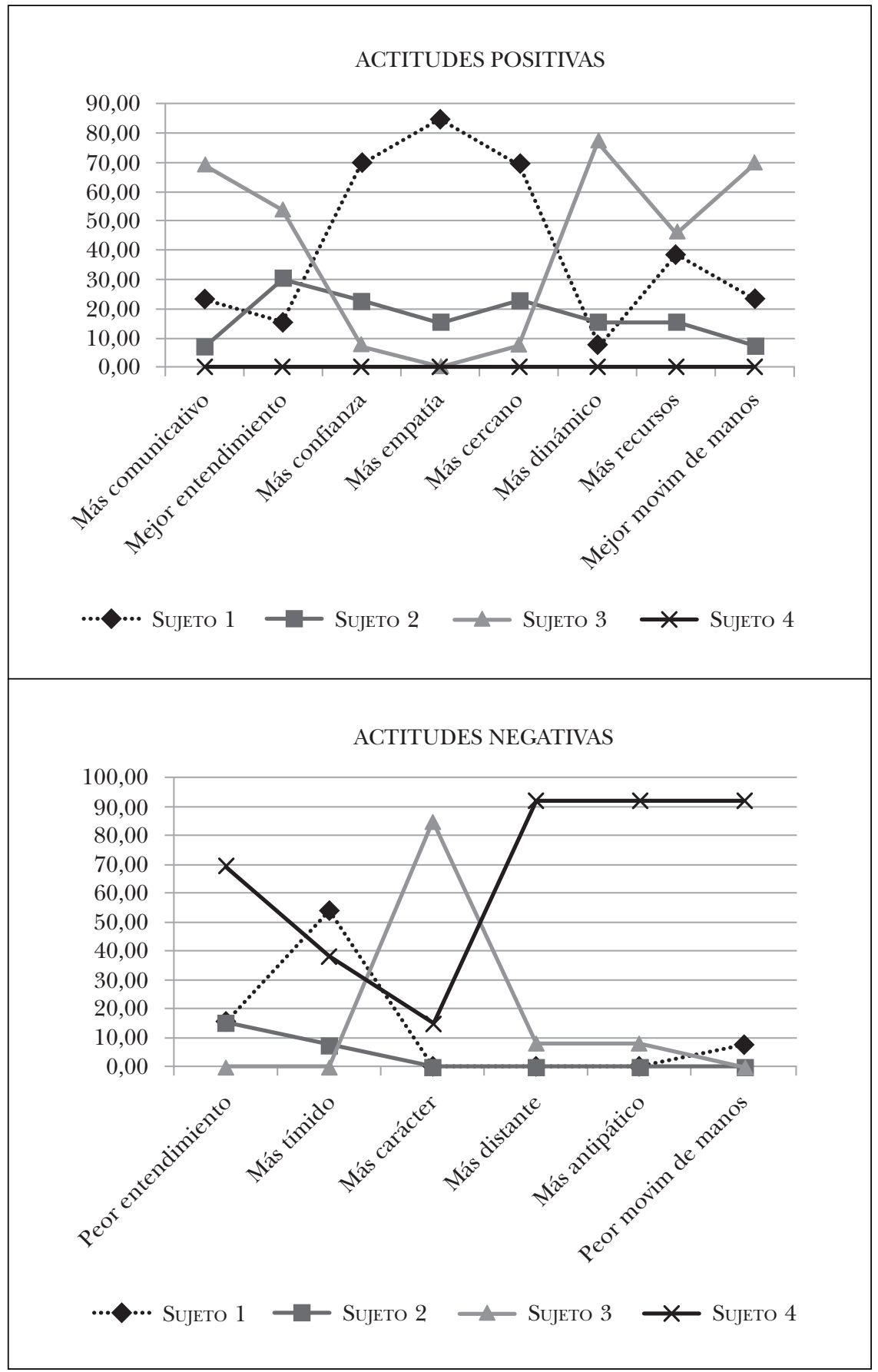


fiable (para el 69,2\% de los estudiantes); más empático $(84,6 \%$ ), y más cercano $(69,2 \%)$. En el tercer puesto encontramos al sujeto 2 y llama la atención que el sujeto 4 no aparece marcado en ninguna de estas variables asociadas a la positividad (de hecho la calificación es de un $0 \%$ en todos los casos).

La otra cara del gráfico, la que muestra las actitudes asociadas a una peor comunicación en el aula relaciona la mayoría de las actitudes negativas con el sujeto 4 (concretamente en cuatro variables de las seis analizadas). El resto de sujetos participantes no destacan en este sentido. Así, el sujeto 4 es el que se percibe por los jóvenes como el que peor se hace entender (así lo estima el $69,2 \%$ del alumnado encuestado), el más distante (92,3\%), el más antipático $(92,3 \%)$ y el que creen que maneja peor sus manos al impartir docencia $(92,3 \%)$. Cabe destacar, no obstante, que el sujeto 1 es el que piensan que es más tímido (al menos para el 53,8\% de los estudiantes) y el sujeto 3 como el que despliega un carácter más fuerte $(84,6 \%)$. Este sujeto 3 es el que presenta porcentajes más bajos en relación a estas actitudes 'poco deseables' en términos de comunicación para la docencia universitaria.

\section{Discusión y CONCLUSIONES}

A modo de conclusión, y tras analizar los resultados del estudio, estamos en condiciones de afirmar que existe una clara relación entre la comunicación no verbal, concretamente el comportamiento kinésico asociado a las manos que despliega el emisor, y la percepción de ciertas actitudes por parte del receptor. De hecho, los datos apoyan numerosos estudios que afirman que la comunicación verbal y no verbal son indisociables y se complementan de forma estrecha.

Yendo a los datos concretos, observamos que aquellos profesores que realizan gestos manuales asociados a la positividad y a la apertura a la comunicación, son definidos por sus alumnos con cualidades relacionadas con el éxito en la transmisión de conocimientos. Es el caso del profesor etiquetado como 'sujeto 3'. El análisis muestra que es el que más tiempo permanece con los brazos abiertos (movimiento asociado a la apertura a la comunicación y a la empatía) y el que no muestra los brazos caídos (dejadez y cansancio), ni cruzados (actitudes defensivas) ni aparece con los puños cerrados (un gesto de agresividad). Por lo que respecta a las manos, presenta una variedad aceptable de movimientos, exhibe las palmas de las manos hacia arriba (transparencia, sinceridad, señal de no tener nada que ocultar), las manos unidas (el único gesto que hay que evitar puesto que simboliza una barrera que impide una comunicación sin obstáculos) 
y enseña los pulgares (asociado a la superioridad). Asimismo, proyecta un repertorio de ilustradores también diverso y en el que predominan los bastones (que ayudan a enfatizar el mensaje), los ideógrafos (que concretan ideas abstractas), los apuntadores (que centran la atención) y los espaciales (que señalan formas y tamaños). Cabe subrayar que precisamente el que obtiene mejores resultados en el análisis objetivo del comportamiento kinésico de las manos es el que se percibe como más comunicativo, el que se hace entender mejor, el más dinámico, con más recursos expresivos y el que mejor mueve las manos. Llama la atención que este 'sujeto 3' en las tres ocasiones en las que no ocupa el primer puesto respecto a los otros tres compañeros lo hace en tercer lugar (confiable, empático y cercano) y nunca en última posición. En cuanto a las actitudes menos deseables en el aula, cabe reseñar que al 'sujeto 3' se le define como el que más carácter tiene. No vuelve a aparecer en los primeros puestos de ninguna actitud asociada a una comunicación poco efectiva. En cambio, es el 'sujeto 4' el que peor sale parado en términos comunicativos. Este docente ocupa siempre el último lugar en las actitudes positivas y el primero de las negativas (es percibido como el que peor se hace entender, el más distante, el más antipático y el que realiza peores movimientos de manos). Es justamente este sujeto el que menos tiempo permaneció con los brazos abiertos (apertura a la comunicación y empatía), el que más los mantuvo bajos (dejadez y cansancio), el único que se frotó las manos (ambición), el que más veces mostró las manos unidas (barrera comunicativa) y las palmas hacia abajo (autoridad y agresividad) y al único que pudo verse con las manos en la espalda (autoridad). Así, aunque sus ilustradores fueron similares a los del resto, permaneció más tiempo que ellos frotándose el rostro (un autoadaptador que se asocia al nerviosismo y a la tensión).

Por lo que respecta a los otros dos docentes, el 'sujeto 1' y el 'sujeto 2', aparecen en los valores medios de la tabla, lo que permite afirmar que ni destacan a la hora de realizar gestos manuales acertados ni son valorados ni como los mejores ni como los peores comunicadores. A pesar de ello, el 'sujeto 1' despliega un repertorio gestual más acertado, un aspecto que hace que se le perciba como el más confiable, el más empático y el más cercano. Asimismo, sus cruces de brazos, la unión de las manos y la fricción del rostro se relacionan directamente con la timidez. Finalmente, el 'sujeto 2' es el que aparece más veces en los valores medios, ocupando la segunda y la tercera posición tanto en los valores positivos como en los negativos. De hecho, se produce idéntica situación a la hora de analizar sus gestos manuales.

Esta investigación aporta profundidad a otras llevadas a cabo por la autora en las que se realizaba un análisis de la comunicación no verbal del emisor para detectar fortalezas y debilidades en el comportamiento kiné- 
sico de personajes públicos, pero que no contrastaba los datos realizando cuestionarios a los receptores. De hecho, el mayor hallazgo reside en la clara relación que se establece entre la proyección de un lenguaje no verbal basado en gestos manuales acertados en términos de éxito comunicativo y la percepción de actitudes positivas en el receptor. Además, aporta luz a la comunicación en la docencia, un campo mucho menos estudiado que otros como la política o la comunicación televisiva. Es importante destacar también que este artículo, al centrarse solo en el comportamiento kinésico de las manos, permite profundizar de forma más exhaustiva en la categorización obteniendo resultados muy precisos. 


\section{BIBLIOGRAFÍA}

Arellano, N. (2006): "Las barreras de la comunicación no verbal entre docentealumno", Revista Orbis, 2, 4, págs. 3-38.

Berganza Conde, Ma R. y Ruiz San Román, J. A. (2005): Investigar en comunicación. Guía práctica de métodos y técnicas de investigación social en comunicación, Madrid, McGraw Hill.

CÁCERES, Mํㅗㄹ D. (2003): Introducción a la comunicación interpersonal, Madrid, Síntesis.

Cruz DíAz, Ma R. (2003): "La comunicación no verbal en contextos educativos. De lo que dicen las miradas", Comunicar. Revista científica de comunicación y educación, 20, págs. 188-194.

EkMAN, P. (1976): "Movements with precise meanings", Journal of Communication, 26, iss. 3, págs. 14-26.

- y FRIESEN W. (1969): "The repertoire of nonverbal behavior: categories, origins, usage, and coding”, Semiotica, 1, iss. 1, págs. 49-98.

Esteve Zaragaga, J. M. (2006): "Las emociones en el ejercicio práctico de la docencia”, Teoría de la Educación, 18, págs. 85-107.

GARCÍA FERNÁNDEZ, J. L. (2000): Comunicación no verbal: periodismo y medios audiovisuales, Madrid, Universitas.

GonzÁlez, J. F. (2003): Lenguaje corporal. El lenguaje mudo del cuerpo, Madrid, Edimat.

Hernández Herrarte, M. y Rodríguez Escanciano, I. (2009): "Investigar en comunicación no verbal: un modelo para el análisis del comportamiento kinésico de líderes políticos y para la determinación de su significación estratégica”, Enseñanza \& Teaching, 27, 1, págs. 61-94.

Mínguez Vela, A. (1999): La otra comunicación. Comunicación no verbal, Madrid, Esic.

Neill, S. y Caswell, C. (2005): La expresión no verbal en el profesorado, Barcelona, Octaedro.

Pease, A. y Pease, B. (2006): El lenguaje del cuerpo, Barcelona, Amat.

Rodríguez Escanciano, I. y Hernández Herrarte, M. (2011): “Análisis de la comunicación no verbal de José Luis Rodríguez Zapatero”, Revista Latina de Comunicación Social, 65, págs. 436-449.

Turchet, P. (2005): El lenguaje de la seducción. Entender los códigos inconscientes de la comunicación no verbal, Barcelona, Amat.

VillahizÁn, J. (2007): Conoce tu personalidad a través del lenguaje corporal, Madrid, Libsa. 


\section{ANEXO \\ Cuestionario para alumnos de $5^{\underline{0}}$ Curso de Publicidad ${ }^{1}$}

Para contestar al cuestionario, encierra en un círculo la alternativa que te parece correcta.

1. ¿Qué profesor te parece que tiene más capacidad de comunicación?
a. Prof. 1
b. Prof. 2
c. Prof. 3
d. Prof. 4

2. ¿A quién de ellos te cuesta menos entender a la hora de explicar conceptos?
a. Prof. 1
b. Prof. 2
c. Prof. 3
d. Prof. 4

3. ¿A quién de ellos te cuesta más entender a la hora de explicar conceptos?
a. Prof. 1
b. Prof. 2
c. Prof. 3
d. Prof. 4

4. ¿¿A quién consideras más tímido?
a. Prof. 1
b. Prof. 2
c. Prof. 3
d. Prof. 4

${ }^{1}$ Este cuestionario es anónimo y forma parte de un estudio cuyo objetivo es el análisis de la influencia del lenguaje de las manos del docente en la asimilación de conceptos por parte del alumnado universitario. 
5. ¿Quién te da más confianza?
a. Prof. 1
b. Prof. 2
c. Prof. 3
d. Prof. 4

6. ¿Quién crees que tiene un carácter más fuerte?
a. Prof. 1
b. Prof. 2
c. Prof. 3
d. Prof. 4

7. ¿Quién te resulta más distante?
a. Prof. 1
b. Prof. 2
c. Prof. 3
d. Prof. 4

8. ¿Quién crees que empatiza mejor con vosotros?
a. Prof. 1
b. Prof. 2
c. Prof. 3
d. Prof. 4

9. ¿Quién te parece más antipático?
a. Prof. 1
b. Prof. 2
c. Prof. 3
d. Prof. 4

10. ¿A quién de ellos consideras más cercano?
a. Prof. 1
b. Prof. 2
c. Prof. 3
d. Prof. 4 
11. ¿Quién te parece más dinámico?
a. Prof. 1
b. Prof. 2
c. Prof. 3
d. Prof. 4

12. ¿Quién crees que utiliza mayor variedad de recursos de aprendizaje (verbales y no verbales)?
a. Prof. 1
b. Prof. 2
c. Prof. 3
d. Prof. 4

13. ¿Quién te parece que mueve mejor las manos a la hora de impartir clase?
a. Prof. 1
b. Prof. 2
c. Prof. 3
d. Prof. 4

14. ¿Quién te parece que mueve peor las manos a la hora de impartir clase?
a. Prof. 1
b. Prof. 2
c. Prof. 3
d. Prof. 4 